\title{
Electrogram Patterns Associated with Successful Radiofrequency Ablation of Accessory Pathways in Children
}

\author{
S.M. Schwartz, ${ }^{1,2}$ M. Dick II, ${ }^{1,2}$ P.C. Dorostkar, ${ }^{1,2}$ G.A. Serwer, ${ }^{1,2}$ S. LeRoy ${ }^{1,2}$ \\ ${ }^{1}$ Division of Pediatric Cardiology, C.S. Mott Children's Hospital, University of Michigan, Ann Arbor, MI 48109-0204, USA \\ ${ }^{2}$ Department of Pediatrics, University of Michigan, Ann Arbor, MI 48109, USA
}

\begin{abstract}
Electrograms observed prior to successful and unsuccessful ablation trials in 33 patients (362 attempts) with manifest pathways and 18 patients (194 attempts) with concealed pathways were compared to identify the electrogram patterns that are associated with successful radiofrequency ablation of accessory atrioventricular connections in young patients (mean age 12.7 years; range 4-22 years). Success was defined as permanent or transient interruption of conduction in the accessory connection. Predictors of success in patients with manifest pathways were local ventricular preexcitation $(p=$ 0.0001 ), left-sidedness (43 or 174) of the accessory connection compared $(p=0.04)$ to right-sidedness (27 of 172 ), a probable Kent bundle potential (29 of 84 versus 39 of $256 ; p=0.0001$ ), and short antegrade atrioventricular conduction intervals $(53.1 \pm 31.9$ ms versus 64.6 $\pm 32.0 \mathrm{~ms} ; p=0.02$ ). Predictors of success in patients with concealed pathways were short ventriculoatrial conduction times $(103.3 \pm 35.8 \mathrm{~ms}$ versus $117.9 \pm 34.8 \mathrm{~ms}$; $p=0.01$ ), and left-sided (42 of 125) pathways ( $p=$ 0.03 ; versus right-sided, 11 of 60 ). The presence of a Kent bundle potential was not significant. We conclude that specific electrogram patterns can predict successful ablation of either manifest or concealed accessory pathways. Use of these criteria may reduce the delivery of unnecessary energy to young myocardium.
\end{abstract}

Key words: Catheter ablation - Child - WolffParkinson-White syndrome - Electrophysiology - Arrhythmias - Supraventricular tachycardia

Use of radiofrequency energy to ablate accessory atrioventricular connections has been shown to be a safe, effective method of treating reentrant supraventricular tachycardia supported by these pathways $[3-5,8-10,12-$ 14, 16-18]. This procedure involves the delivery of ra-

Correspondence to: M. Dick II diofrequency energy to target myocardial tissue at or immediately adjacent to the mapped accessory connection, producing coagulation necrosis of myocardial cells [1]. The ability to predict successful ablation from endocardial bipolar electrogram characteristics would in all likelihood reduce the number of radiofrequency energy applications as well as the duration of the procedure. The purpose of this study therefore was to determine if there are characteristic electrogram patterns predictive of successful ablation of manifest or concealed accessory connections in young patients. We postulated that because of the necessarily close juxtaposition of the ablating catheter and the accessory connection successful termination of retrograde conduction would be related to the amplitude of the atrial and ventricular electrograms measured through the ablating catheter, the conduction intervals (both antegrade and retrograde) measured through the ablating catheter, and the presence of a Kent bundle potential recorded by the ablating catheter.

\section{Materials and Methods}

Beginning in April 1990 (when our radiofrequency ablation program was initiated) through April 1992 available data from 45 consecutive young patients who underwent electrophysiologic study with radiofrequency ablation (52 accessory connections) were collected. A group of 27 children had a single manifest accessory connection (age 4-22 years, mean 12.8 years; 22 males, 11 females), and 12 children had a total of 13 concealed accessory connections (age 4-19 years, mean age 12.7 years; 14 males, 4 females). Six patients had a single manifest and a single concealed accessory connection each. Electrophysiologic study revealed that 19 of the manifest pathways were left-sided and 14 were right-sided. Of the concealed pathways, 13 were left-sided and 6 were right-sided. Informed consent for electrophysiologic study and radiofrequency ablation was obtained from the parents or from the patient when age-appropriate. The protocol for radiofrequency ablation in children was approved by the Institutional Review Board of the University of Michigan.

Methods for electrophysiologic study and radiofrequency ablation of accessory connections in our laboratory have been previously described [4]. Atrial and ventricular bipolar electrograms were re- 
corded (Siemens Mingo 7, paper speed 100-200 mm/s, band width 50-500 Hz) through electrodes (Mansfield 7F steerable quadripolar catheter, no. 5250) and through electrodes positioned along the atrioventricular groove within the coronary sinus (Mansfield orthogonal electrode catheter, no. 5211). Right-sided accessory connections were mapped with the ablation catheter advanced into the right atrium through the right femoral vein and inferior vena cava. Left-sided pathways were mapped with the ablation catheter inserted through the right femoral artery and passed retrogradely across the aortic valve. Under fluoroscopic guidance the steerable catheter tip was then positioned under the mitral valve leaflets. In one patient with a left-sided accessory connection, the ablation catheter was placed in the left atrium across the atrial septum by means of a transseptal puncture. Radiofrequency current (Radionics, RFG-3C lesion generator system) was delivered during normal sinus rhythm, supraventricular reentrant tachycardia, ventricular pacing at $40-70$ volts for $2-60$ seconds. At $0.5-1.0$ hour after delivery of radiofrequency energy, evidence of retrograde conduction and inducible reentrant tachycardia was sought by atrial and ventricular pacing and extra stimulation.

To determine potential predictors of successful ablation, antegrade atrioventricular conduction intervals in patients with manifest pathways and retrograde conduction intervals in patients with either manifest or concealed pathways were measured. Intervals (milliseconds) and amplitudes (millivolts) were measured via the electrograms recorded through the distal pair of electrodes on the ablating catheter unless otherwise specified. The intervals were the AV interval, measured from the onset of atrial activation to the onset of ventricular depolarization; the A-V interval (isoelectric interval), measured from the end of atrial activation to the beginning of ventricular activation; and the QA interval, measured from the onset of ventricular activation in any lead to the first rapid deflection in the atrial electrogram. In addition, because the retrograde conduction intervals were obtained during supraventricular tachycardia (SVT), ventricular pacing, or both, we determined the isoelectric VA interval, measured from the end of ventricular depolarization to the beginning of atrial depolarization. This measurement, it was judged, could possibly offset the differences in intraventricular conduction time present during SVT and ventricular pacing. Finally, because the mapping catheters (and electrodes), in contrast to the movable ablating catheter (and electrodes), were stationary and thus might not be closest to the accessory connection, the A-A ${ }^{\prime}$ interval (Fig. 1) was evaluated during retrograde conduction in patients with concealed pathways. This parameter was defined as the interval from atrial activation at the ablating electrode to atrial activation at the reference electrode along the atrioventricular groove closest to the accessory connection and was measured so a negative value indicated atrial activation at the ablating electrode prior to atrial activation at the reference electrode. Amplitudes of the atrial and ventricular electrograms were measured in millivolts between the points of maximal excursion.

Tracings were scrutinized for possible Kent bundle potentials as indicated by a high-frequency low-amplitude signal not coincident with the $\mathrm{P}$ wave or the QRS complex during sinus rhythm (in manifest pathways) or SVT, or by continuous electrical activity between the atrial and ventricular electrograms (Fig. 2). Displacement of the atrial electrogram from the suspect Kent potential by atrial extrastimulation during SVT or ventricular pacing was not frequently used to confirm the suspect potential as a Kent potential.

Local ventricular preexcitation, that is, the interval measured from the onset of ventricular activity recorded through the distal pair of electrodes of the ablating catheter and the onset of the surface QRS complex during sinus rhythm (Fig. 3), was determined. The interval (seconds) from onset of radiofrequency current to disruption of retrograde conduction in successful attempts was measured.

Success was defined as permanent or transient interruption of
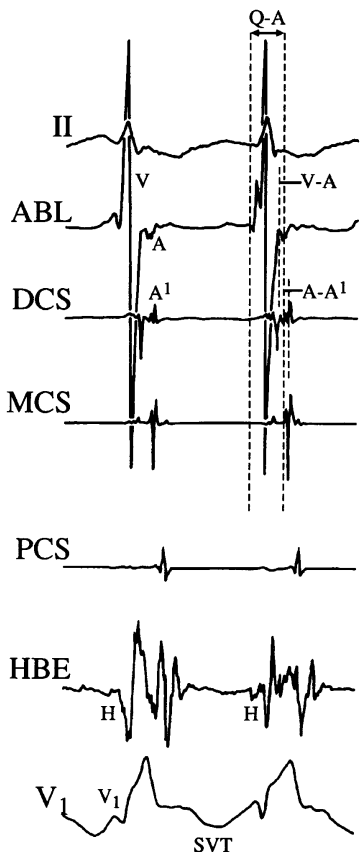

$100 \mathrm{~mm} / \mathrm{sec}$

Fig. 1. Retrograde conduction intervals measured in patients with concealed accessory connections. The Q-A interval is measured from the earliest onset of ventricular activation in any lead to the onset of atrial activation at the distal electrode pair of electrodes on the ablating catheter. The V-A interval is measured from the end of the ventricular electrogram to the onset of the atrial electrogram recorded through the distal electrode pair of the ablating catheter. The A- $\mathrm{A}^{1}$ interval is measured from the onset of atrial activation at the distal electrode pair of the ablating catheter to the onset of atrial activation through the electrode pair of the mapping catheter closest to the accessory connection. $A$, atrial electrogram recorded at the distal electrode pair of the ablating catheter; $A^{l}$, atrial electrogram recorded at the mapping electrode closest to the accessory connection; $A B L$, distal electrode pair electrogram of the ablating catheter; $D C S$, distal coronary sinus electrogram; $H$, His bundle potential electrogram; $H B E$, His bundle electrogram; $M C S$, midcoronary sinus electrogram; $P C S$, proximal coronary sinus electrogram; $S V T$, supraventricular tachycardia; $V$, ventricular electrogram recorded at the ablating electrode through the distal electrode pair of the ablating catheter; $V_{1}$, electrocardiographics lead $V_{1}, I I$, electrocardiographics lead II.

conduction in the accessory connection. Characteristics of successful and unsuccessful attempts in both manifest and concealed pathways are reported, when continuous variables, as the mean $\pm 1 \mathrm{SD}$ and are compared using the one-tailed Student's $t$-test; categorical variables were examined using the chi-square analyses. A $p$ value of $\leqslant 0.05$ was taken to denote statistical significance.

\section{Results}

A total of 556 radiofrequency trials were delivered, with a range of 1-37 per study. Data (two manifest and three concealed) prior to five radiofrequency ablation trials 


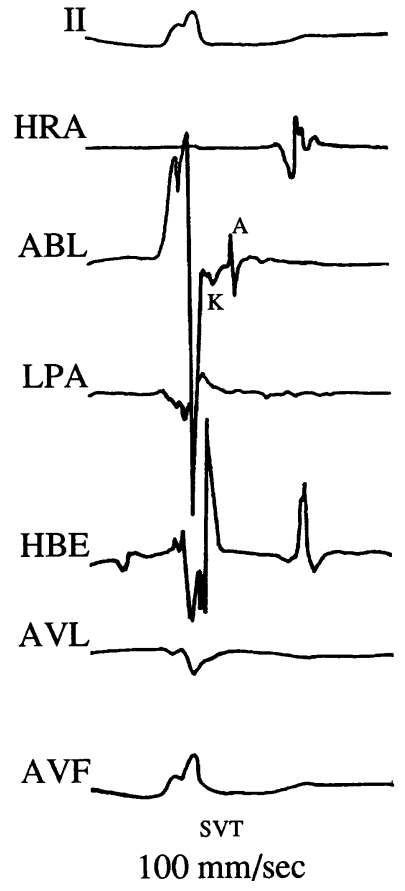

Fig. 2. Example of a possible Kent potential recorded through the two distal electrodes on the ablating catheter during retrograde conduction while in SVT from a patient with a manifest accessory connection. $A$, atrial electrogram; $A B L$, ablating electrode; $A V F$, unipolar limb lead on left leg; $A V L$, unipolar limb lead on left arm; $H B E$, His bundle electrogram; $H R A$, high right atrial electrogram; $K$, Kent potential; $L P A$, left atrial appendage electrogram; $S V T$, supraventricular tachycardia.

were unavailable for evaluation; and in several tracings only a few variables could be measured. A total of 362 trials were in patients with manifest (1-37) accessory connections. In these patients 70 trials successfully interrupted conduction through the accessory connection: 43 transient, 27 permanent. Thirty-six transient ablations eventually resulted in permanent ablation. There were 262 unsuccessful trials. In those patients with concealed accessory connections, there were 194 trials (1-30); 53 were successful: 41 transient, 12 permanent. Eighteen of the transient ablations were followed by permanent ablation; 126 attempts were unsuccessful. Possible Kent potentials were present in 84 of 340 tracings in patients with preexcitation and in 63 of 171 tracings from patients with concealed accessory connections.

\section{Manifest Pathways}

Of the 33 patients with preexcitation, 27 had successful permanent ablation of their accessory connections $(82 \%)$.

Successful Predictors. Successful trials were most strongly marked by ventricular activity recorded through

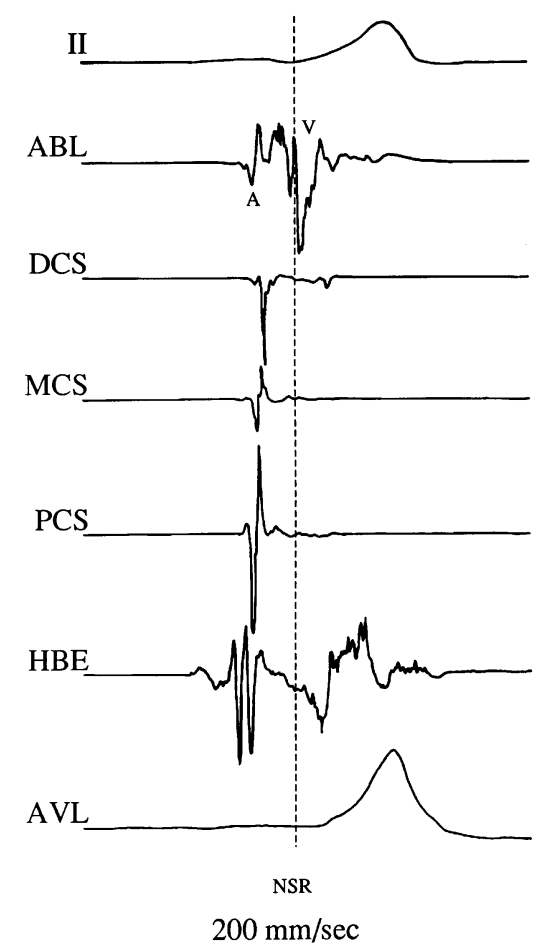

Fig. 3. Local ventricular preexcitation. Ventricular activation is recorded through the distal pair of electrode on the ablating electrode prior to the onset of the delta wave (dashed line) on the surface electrocardiogram in a patient with a manifest accessory connection. A, atrial electrogram recorded at the ablating electrode; $A B L$, ablating electrode; $D C S$, distal coronary sinus electrogram; $H B E$, His bundle electrogram; $M C S$, midcoronary sinus electrogram; NSR, normal sinus rhythm; $P C S$, proximal coronary sinus electrogram; $V$, ventricular electrogram recorded at the ablating electrode.

the ablating electrode that preceded the onset of the QRS complex on the surface electrocardiogram (ECG) during sinus rhythm (local ventricular preexcitation) (Fig. 3). In successful attempts, local ventricular preexcitation was identified at the ablating electrode $12.6 \pm 1.5 \mathrm{~ms}$ prior to the QRS complex, compared to $1.2 \pm 1.1 \mathrm{~ms}$ in unsuccessful trials $(p=0.0001)$. When ventricular activation at the ablating electrode preceded the QRS complex by $>15 \mathrm{~ms}, 17$ of $53(32 \%)$ of trials were successful, compared to 17 of $184(9 \%)$ when this interval was $\leqslant 15 \mathrm{~ms}$. Trials to ablate left-sided accessory connections $(25 \%$; 43 of 174) were likewise more successful $(p=0.04)$ than attempts to disrupt right-sided pathways $(16 \% ; 27$ of 172). Identification of electrical activity suggestive of a Kent bundle potential $(35 \%, 29$ of 84 versus $15 \%, 39$ of 256) was also associated $(p=0.001)$ with an increased likelihood of a successful outcome. Finally, the antegrade conduction interval measured from the onset of atrial activation to the onset of ventricular activation (AV, Table 1) was shorter during successful attempts by an average of $>10 \mathrm{~ms}$. In 36 of $40(90 \%)$ successful trials in which the AV interval was measured, the value was 
Table 1. Conduction intervals versus outcome in manifest pathways

\begin{tabular}{|c|c|c|c|c|}
\hline Condition & $\mathrm{AV}(\mathrm{ms})$ & $\mathrm{A}-\mathrm{V}(\mathrm{ms})$ & QA (ms) & VA (ms) \\
\hline Successful & $53.1+31.9$ & $14.3+15.0$ & $110.2+28.4$ & $10.1+6.9$ \\
\hline Unsuccessful & $64.6+32.0$ & $18.0+16.3$ & $106.0+33.3$ & $12.4+14.8$ \\
\hline Significance $(p)$ & $p=0.02$ & NS & NS & NS \\
\hline
\end{tabular}

$\mathrm{AV}$, onset of atrial activation to onset of ventricular depolarization (ablating electrode); $\mathrm{A}-\mathrm{V}$, end of atrial activation to the onset of ventricular depolarization (isoelectric interval) (ablating electrode); QA, onset of ventricular activation in any lead to first rapid deflection of the atrial electrogram at the ablating electrode; VA, end of ventricular depolarization to onset of atrial activation retrograde (isoelectric VA) at the ablating electrode.

$<80.5 \mathrm{~ms}$; only 34 of 156 trials $(22 \%)$ in which the AV interval was less than $80.5 \mathrm{~ms}$ resulted in ablation of the pathway.

Unsuccessful Predictors. There was no significant difference in the A-V interval or retrograde conduction intervals (Table 1) between those trials that terminated preexcitation and those that did not. There was no significant relation between the atrial or ventricular amplitudes during either sinus rhythm or SVT/ventricular pacing.

Successful Ablation Interval. The average time to cessation of preexcitation or retrograde conduction in those trials that were successful was $5.0 \pm 3.9$ seconds. Permanent ablation occurred within a significantly ( $p=$ $0.005)$ shorter $(3.4 \pm 2.2$ seconds) interval after onset of radiofrequency energy than did transient ablation $(6.1 \pm$ 4.5 seconds). A total of $95 \%$ of ablations occurred within 12 seconds of energy application for all disruptions of the accessory connection and within 6 seconds for permanent ablation.

\section{Concealed Pathways}

Of the 19 pathways in these 18 patients, 12 were permanently ablated $(63 \%)$. Ablation procedures in patients with concealed pathways were not as frequently successful as those in patients with manifest pathways, although this difference was not statistically significant $(p=$ 0.08 ). Of the 19 concealed accessory connections, 13 were left-sided.

Successful Predictors. Similar to those patients with manifest pathways, ablation attempts directed at leftsided pathways $(34 \% ; 42$ of 125$)$ were more frequently successful $(p=0.03)$ than ablation of right-sided pathways $(18 \% ; 11$ of 60$)$. Unlike our experience in patients with preexcitation, the presence of electrical activity suggestive of a Kent bundle potential did not enhance success of ablation attempts in patients with concealed accessory connections $(32 \%, 20$ of 63 versus $21 \%, 28$ of 136). Retrograde conduction intervals measured in these patients also demonstrated a statistically significant difference between those trials that interrupted retrograde conduction in the accessory pathway and those that did
Table 2. Conduction intervals versus outcome in concealed pathways

\begin{tabular}{llcl}
\hline \multicolumn{1}{c}{ Condition } & QA $(\mathrm{ms})$ & $\mathrm{A}-\mathrm{A}^{\prime}(\mathrm{ms})$ & $\mathrm{VA}(\mathrm{ms})$ \\
\hline Successful & $103.3+35.8$ & $-10.1+12.8$ & $11.7+15.5$ \\
Unsuccessful & $117.9+34.8$ & $-2.7+21.1$ & $14.3+18.1$ \\
Significance $(p)$ & $p=0.01$ & $p=0.01$ & NS \\
\hline
\end{tabular}

QA, onset of ventricular activation in any lead to first rapid deflection of the atrial electrogram at the ablating catheter; A-A', onset of atrial activation at the ablating electrode to earliest atrial activation in mapping catheter retrograde; VA, end of ventricular depolarization to onset of atrial activation retrograde in the ablating catheter.

not (Table 2). Evaluation of the conduction intervals reveals that the time between the onset of ventricular depolarization and the atrial electrogram (QA interval) was shorter in successful ablation attempts $(103.3 \pm 35.8 \mathrm{~ms})$ than in unsuccessful trials $(117.9 \pm 34.8 \mathrm{~ms} ; p=0.01)$. The $\mathrm{AA}^{\prime}$ interval was an average of $7 \mathrm{~ms}$ shorter in those trials resulting in cessation of retrograde conduction in the accessory connection, indicating that atrial activation at the ablating electrode was earlier than at the mapping electrode. Only 1 of 58 ablation attempts in which the $\mathrm{AA}^{\prime}$ interval was $>0$ resulted in success, yielding a negative predictive value of $97 \%$ ( $p=0.0001)$.

Unsuccessful Predictors. The VA interval (measured from the end of ventricular depolarization to the beginning of atrial depolarization, the isoelectric VA interval) was unrelated to successful outcome in patients with concealed pathway(s). Similar to the findings in patients with manifest pathways, amplitudes of the atrial and ventricular electrograms did not predict a successful interruption of the accessory connection.

Successful Ablation Interval. Successful response was marked by a prompt response with a mean time of $5.4 \pm 3.8$ seconds. Permanent ablation $(3.7 \pm 2.0 \mathrm{sec}-$ onds) tended to occur more promptly $(p=0.06)$ than did transient ablation $(6.0 \pm 4.1$ seconds). A total of $95 \%$ of all ablations occurred in $<11.7$ seconds, whereas all permanent ablations occurred in $<8.1$ seconds, and $91 \%$ in $<6$ seconds.

Age and Body Size. Grouping of all patients by age and size failed to detect a relation between these two 
variables and the likelihood of success of the radiofrequency trials.

\section{Discussion}

These data indicate that factors associated with a successful outcome of radiofrequency ablation of accessory connections in young patients with preexcitation are local ventricular preexcitation, a possible Kent potential, and an electrogram pattern recorded through the ablating catheter's distal pair of electrodes marked by a short AV conduction interval, as measured from the onset of atrial activation to the onset of ventricular activation. In patients with concealed accessory connections, electrogram patterns at the distal pair of electrodes on the ablating catheter during SVT or ventricular pacing that were predictive of success were the retrograde QA interval (measured from the onset of ventricular activation on the surface ECG to the onset of the atrial electrogram at the ablating electrode) and the occurrence of atrial activation earlier at the ablating electrode than at any of the mapping electrodes. Localization to the left side of the heart was associated with greater likelihood of successful outcome in both manifest and concealed pathways. Finally, a successful response was prompt, suggesting the strategy of limiting the duration of discharge to 6-10 seconds.

There is widespread agreement regarding successful predictors of successful ablation of manifest pathways. Kuck and Schluter reported predictors similar to ours for successful ablation of left-sided manifest pathways using a single retrograde catheter for mapping and energy delivery in 34 young patients. Sites for ablation were selected by the presence of electrical activity suggestive of a Kent potential. The ablation procedure was successful in $88 \%$ of their patients with left-sided pathways and an identifiable accessory connection potential [11]. The antegrade AV interval measured through the ablating electrode in sinus rhythm was shorter in successful trials, although most trials in which the AV interval was $<50$ ms failed to eliminate preexcitation [11]. Similar to our findings, these authors also found no relation between the amplitude of the atrial and ventricular electrograms and the likelihood of a successful outcome of a given ablation trial. Haissaguerre and colleagues [7] studied 135 patients with manifest accessory connections and also found that the only predictor of outcome was a shorter antegrade conduction time. They found no statistically significant predictors of success among retrograde conduction times or amplitude of atrial and ventricular electrograms. Calkins et al. [2] found in their patients with manifest pathways that successful outcome was related to the presence of a Kent potential and to the occurrence of local ventricular preexcitation.

In agreement with a report of Grimm and Josephson
[6] regarding patients with concealed pathways, shorter retrograde (QA and $\mathrm{AA}^{\prime}$ ) conduction intervals were predictive of successful ablation. In contrast, a suspect Kent potential failed to predict successful ablation in our patients with concealed pathways, an observation that was not only opposite to our findings and to those of Kuck and Schluter in patients with preexcitation but also to those of Calkins et al. in patients with concealed pathways. This unexpected finding probably arises from lack of verification of the suspect Kent potential as well as technical differences such as in filters and amplification among the various studies.

No correlation was found between the amplitudes of the atrial and ventricular electrograms and the likelihood of success in our study and those of others $[2,6,7,11]$. This finding indicates that the relative amplitudes of the atrial and ventricular electrograms are informative only regarding the proximity to the atrioventricular groove; they do not necessarily indicate the proximity to the accessory connection. As a result, electrogram amplitude is a poor predictor of successful ablation.

Successful outcome in patients who have manifest or concealed accessory connections was characterized by abrupt cessation of retrograde conduction after the initiation of energy delivery, similar to the findings of other investigators [11]. Permanent ablation occurred more promptly than did transient ablation. These data indicate that when the ablating electrode is appropriately positioned relative to the accessory pathway and in contact with the target tissue sufficient radiofrequency energy is deliver within a short time to disrupt the accessory connection, its origin, or its insertions. The failure of interruption of anomalous conduction within 6-10 seconds of radiofrequency energy strongly suggests that the tip electrode of the ablating catheter may not be in immediate proximity to the accessory connection, or alternatively, not be abutting the target tissue sufficiently to achieve the necessary temperature to disrupt the AV connection. Temperature-sensitive catheters monitoring this variable may overcome this disadvantage.

Of interest would be the detection of age- and sizerelated differences in the prediction of successful ablation of accessory connections in children. Because the general lesion size is independent of the mass and dimensions of the heart, one could postulate that, all other factors being equal [1], the same energy level delivered to a child as delivered to an adult would result in a relatively larger lesion and thus would be more likely to include the origin or insertion of the accessory connection within it. One could further postulate that similar electrophysiologic measures suggest a closer proximity between the targeted accessory connection and the ablating electrode, yielding a more likely chance of success. However, no age- or size-related differences were detected among our patients aged 3-20 years. This unexpected finding suggests that individual intracavitary features of the myocardium as well as technical limita- 
tions of catheter placement play a significant role in determining successful ablations [15].

A number of criteria are associated with an increased likelihood of interruption of accessory atrioventricular pathways with radiofrequency energy in young patients: maximal local ventricular preexcitation (manifest pathways), shortest achievable conduction intervals, suspect Kent potentials (manifest pathways), and prompt response to energy application. Adherence to these criteria may reduce the delivery of unnecessary radiofrequency energy to young myocardium.

\section{References}

1. Borggrefe M, Hindricks G, Haverkamp W, Breithardt G (1990) Catheter ablation using radiofrequency energy. Clin Cardiol 13: 127-131

2. Calkins H, Kim YN, Schmaltz S, et al (1992) Electrogram criteria for identification of appropriate target sites for radiofrequency catheter ablation of accessory atrioventricular connections. Circulation 85:565-573

3. Calkins H, Sousa J, El-Atassi R, et al (1991) Diagnosis and cure of the Wolff-Parkinson-White syndrome or paroxysmal supraventricular tachycardias during a single electrophysiologic test. $N$ Engl $J$ Med 324:1612-1618

4. Dick M, O’Connor BK, Serwer GA, LeRoy S, Armstrong B (1991) Use of radiofrequency current to ablate accessory connections in children. Circulation 84:2318-2324

5. Evans GT, Huang WH, CAR Investigators (1990) Catheter ablation of accessory atrioventricular pathways: early results of a prospective international multicenter study. Circulation 82(Suppl III): 690 [abstract]

6. Grimm W, Josephson ME (1993) Comparison of successful and unsuccessful sites of radiofrequency catheter ablation of concealed accessory AV-connections. Circulation 88(Suppl I):63 [abstract]

7. Haissaguerre M, Dartigues J-F, Warin J-F, et al (1991) Electrogram patterns predictive of successful catheter ablation of acces- sory pathways: value of unipolar recording mode. Circulation 84 : 188-202

8. Jackman WM, Wang X, Friday KJ, et al (1991) Catheter ablation of accessory atrioventricular pathways (Wolff-Parkinson-White syndrome) by radiofrequency current. $N$ Engl J Med 324:16051611

9. Jackman W, Wang X, Moulton K, et al (1990) Role of the coronary sinus in radiofrequency ablation of left free-wall accessory AV pathways. Circulation 82(Suppl III):689 [abstract]

10. Kuck KH, Kunze KP, Geiger M, Schluter M (1989) Radiofrequency ablation of accessory atrioventricular pathways. Circulation 80(Suppl II):323 [abstract]

11. Kuck KH, Schluter M (1991) Single-catheter approach to radiofrequency current ablation of left-sided accessory pathways in patients with Wolff-Parkinson-White syndrome. Circulation 84: 2366-2375

12. Kuck KH, Schluter M, Geiger M, Siebels J, Duckeck W (1990) Radiofrequency current approach to successful catheter ablation of accessory pathways. Circulation 82(Suppl III):689 [abstract]

13. Morady F, Kadish A, Calkins H, et al (1990) Diagnosis and immediate cure of paroxysmal supraventricular tachycardia. Circulation 82(Suppl III):689 [abstract]

14. Roman CA, Friday KJ, Wang X, et al (1989) Ablation of single and multiple accessory pathways with radiofrequency current. Circulation 80 (Suppl II):323 [abstract]

15. Saul JP, Hulse JE, De W, et al (1993) Catheter ablation of accessory atrioventricular pathways in young patients: use of long vascular sheaths, the transseptal approach and a retrograde left posterior parallel approach. J Am Coll Cardiol 21:571-583

16. Saul JP, Walsh EP, Langberg JJ, Mayer JE, Lock JE (1990) Radiofrequency ablation of accessory atrioventricular pathways: early results in children with refractory SVT. Circulation 82(Suppl III): 222 [abstract]

17. Scheiman MM, Laks MM, DiMarco J, Plumb V (1991) Current role of catheter ablative procedures in patients with cardiac arrhythmias: a report for the health professionals from the Subcommittee on Electrocardiography and Electrophysiology, American Heart Association. Circulation 83:2146-2153

18. Schluter M, Geiger M, Siebels J, Duckeck W, Kuck KH (1991) Catheter ablation using radiofrequency current to cure symptomatic patients with tachyarrhythmias related to an accessory atrioventricular pathway. Circulation 84:1644-1661 